Meta

Journal des tradlucteurs

Translators' Journal

\title{
Incidence de la langue juridique sur la langue de la gestion
}

Robert Dubuc

Volume 24, numéro 1, mars 1979

La traduction juridique

URI : https://id.erudit.org/iderudit/002597ar

DOI : https://doi.org/10.7202/002597ar

Aller au sommaire du numéro

Éditeur(s)

Les Presses de l'Université de Montréal

ISSN

0026-0452 (imprimé)

1492-1421 (numérique)

Découvrir la revue

Citer cet article

Dubuc, R. (1979). Incidence de la langue juridique sur la langue de la gestion.

Meta, 24(1), 159-165. https://doi.org/10.7202/002597ar d'utilisation que vous pouvez consulter en ligne.

https://apropos.erudit.org/fr/usagers/politique-dutilisation/ 


\section{Incidence de la langue juridique sur la langue de la gestion}

Dans le fonctionnement quotidien de l'entreprise, les réalités juridiques occupent une place d'une certaine importance que reflète, bien sûr, le vocabulaire courant de la gestion.

Il y a d'abord les expressions qui relèvent des affaires juridiques de l'entreprise : relations avec les tribunaux, poursuites, contrats, droits. Viennent ensuite les expressions reliées à la structure juridique et à l'exploitation de l'entreprise. $\AA$ ces questions, on peut rattacher certaines expressions utilisées dans les assemblées délibérantes. Enfin, il circule bon nombre d'expressions courantes à coloration juridique.

Il ne saurait être question, dans le cadre d'un article comme celui-ci, de traiter ces diverses facettes du vocabulaire juridique d'une façon exhaustive. Nous allons tâcher de retenir, à l'intérieur de chaque catégorie, quelques expressions qui posent des problèmes aux traducteurs et terminologues d'entreprises.

\subsection{Affaires juridiques ${ }^{2}$}

La traduction de l'adjectif legal entraîne des difficultés. Ces problèmes ne sọnt pas nouveaux. Tous les chroniqueurs linguistiques ont à tour de rôle épilogué sur cette question. L'erreur la plus courante est évidemment de traduire automatiquement legal par LEGAL.

Le service qui s'occupe des affaires juridiques d'une entreprise se nomme en anglais legal department d'où les traductions de « département légal » ou de " service légal s. En français deux appellations sont pour ainsi dire en concurrence CONTENTIEUX ${ }^{2}$ et SERVICES JURIDIQUES. Ordinairement, le contentieux ne s'occupe que des affaires litigieuses, tandis que les services juridiques prennent à leur charge l'ensemble des affaires juridiques, y compris la gestion des contrats, l'application des lois, etc.

Les services juridiques sont habituellement dirigés par un CONSEILLER JURIDIQUE (legal advisor), improprement nommé « aviseur légal ». Il peut être

1. A moins de mention contraire, les equivalents proposés sont tirés des fiches du Comité

2. Paul Robert, Dictionnaire alphabétique et analogique de la langue française, Paris, Société du Nouveau Littre, $1960+$, sub verbis. 
assisté d'une secrétaire spécialisée dans ce genre de travail (legal secretary) qu'on nomme SECRÉTAIRE JURIDIQUE.

Si le conseiller juridique ne travaille pas à plein temps pour l'entreprise, il est souvent rémunéré au moyen d'un retainer fee, sorte d'honoraires forfaitaires garantis, dont on défalque par la suite les autres honoraires à verser pour les divers services rendus. Ce retainer fee s'appelle en français une PROVISION.

$\begin{array}{lll}\begin{array}{l}\text { Anglais } \\ \text { legal department }\end{array} & \begin{array}{l}\text { Usage fautif } \\ \text { « département légal » }\end{array} & \begin{array}{l}\text { Français } \\ \text { contentieux } \\ \text { service(s) juridiques(s) }\end{array} \\ \text { legal advisor } & \text { "aviseur légal » } & \begin{array}{l}\text { conseiller juridique } \\ \text { legal secretary }\end{array} \\ \text { retainer fee } & \text { secrétaire légal(e) } & \text { secrétaire juridique }\end{array}$

Dans ses relations avec les tribunaux, l'entreprise utilise les expressions courantes du milieu. On relève souvent des termes comme «mépris de cour», « libelle », «sub poena ». On reconnaît là de vieux anglicismes que le conservatisme de nos gens de robe rend bien difficiles à déraciner.

\begin{tabular}{|c|c|c|}
\hline $\begin{array}{l}\text { contempt of court } \\
\text { libel }\end{array}$ & $\begin{array}{l}\text { "mépris de cour» } \\
\text { «libelle» }\end{array}$ & $\begin{array}{l}\text { outrage au tribunal } \\
\text { diffamation }\end{array}$ \\
\hline & $\begin{array}{l}\text { Se faire intenter une } \\
\text { action en libelle. }\end{array}$ & $\begin{array}{l}\text { Se faire intenter un } \\
\text { procès en diffamation. }\end{array}$ \\
\hline sub poena & ¿ sub poena & citation à comparaître $^{s}$ \\
\hline
\end{tabular}

Souvent l'usage québécois et canadien tend à conserver telles quelles les expressions juridiques anglaises empruntées au latin ou à l'ancien français. Ainsi le mot lien, d'origine française, s'emploie ici au sens de DROIT DE RETENTION, CHARGE. «Une propriété tibre de tout lien » devrait se dire : «libre de toute charge ou servitude *

lien

\& lien $\quad$\begin{tabular}{lll}
$\quad$ & $\begin{array}{l}\text { droit de rétention } \\
\text { charge }\end{array}$ \\
\hdashline & & servitude
\end{tabular}

Les services juridiques sont appelês à faire la distinction, au moins sommaire, entre FAILLITE et BANQUEROUTE. Il ne s'agit pas d'entrer dans des subtilités juridiques, mais bien de discerner la portée générale de ces termes. La banqueroute est accompagnée d'actes délictueux. La faillite est un acte absolument légal 5 .

3. J. Jéraute, Vocabulaire français-anglais, anglais-français de termes et locutions juridiques, Paris, Librairie générale de Droit et de Jurisprudence, 1953, sub verbis.

4. H. Carbonneau, Vocabulaire général anglais-français, Ottawa, Secrétariat d'Etat, 1972 ,

7 vol., sub verbis.

5. Paul Robert, Dictionnaire alphabêtique et analogigue de la langue française. 
Le mot anglais bankrupt peut avoir les deux sens. Il s'agit de ne pas les confondre.

legal bankruptcy

faillite

fraudulent bankruptcy

banqueroute

La gestion des contrats relève du service juridique, mais leur traduction est habituellement confiée, cela va de soi, au traducteur qui s'arrache littéralement les cheveux devant les redondances, les énumérations, les charnières archaïques de la langue juridique anglaise. $\mathrm{La}$ redondance la plus courante nous est fournie par l'expression terms and conditions, qu'il suffit de rendre en français par CONDITIONS.

terms and conditions \& termes et conditions \ conditions

Les contrats utilisent en outre un certain nombre de formules stéréotypées dont la traduction n'est pas toujours orthodoxe.

party of the first part \& partie de première part " d'une part

party of the second part \& partie de seconde part " d'autre part

in witness thereof

en foi de quoi

hereinafter called

ci-après appelé

whereas

attendu que?

wherein

(à rendre par les pro-

whereof noms conjonctifs pertinents : où, dont, duquel, etc.)

Il y a de plus, lorsqu'on énonce une alternative dans un contrat, une formule largement utilisée whichever comes first lorsqu'il s'agit d'événements, whichever shall be less, ou is the greater, lorsqu'il s'agit de montants.

whichever comes first

whichever shall be less

whichever is the greater selon la première éventualité

selon le montant le plus bas

selon le montant le plus élevé.

Dans les contrats commerciaux, il est souvent question de royalties, de licensing et d'assignment of rights.

Pour royalties le problème se trouve résolu par REDEVANCE \&, expression proposée par les arrêtés de terminologie de la République française. Le licensing consiste à donner à un tiers le droit de fabriquer les produits du licensor.

6. H. Carbonneau, Vocabulaire général anglais-français.

7. J. Jéraute, Vocabulaire français-anglais, anglais-français de termes et locutions juri-

8. Arrêtés de terminologie de la République française, publiés par le Bureau des traductions, Bulletin de terminologie no 155 , Ottawa, Secrétariat d'Etat, 1974. 
licensing

(fabrication/production) sous licence ${ }^{8}$

licensor

concédant (n.m.) ${ }^{10}$

licensee

titulaire d'une licence ${ }^{11}$

license-holder

Enfin le contrat peut prévoir deux types d'abandon de droits : l'assignment of rights et la waiver. Dans le premier cas, il s'agit de la oession d'un ou de plusieurs droits, dans le second d'une renonciation à l'exercice d'un droit.

assignment of (a) right(s)

cession d'un droit ${ }^{12}$

waiver

renonciation ${ }^{13}$

Une fois le contrat terminé, il faudra en établir des copies qui, pour faire foi, devront être authentifiées. On dira en aglais certified true copy et en français COPIE CERTIFIEE CONFORME.

certified true copy

copie certifiée conforme ${ }^{14}$

\subsection{Assemblées délibérantes}

Dans un deuxième volet, ce sont quelques termes relatifs à la "procédure » des assemblées délibérantes qui vont retenir notre attention. Nous touchons ici à un secteur parajuridique, si l'on peut dire, mais le caractère officiel que prennent certaines assemblées, en particulier l'assemblée générale des actionnaires, n'exclut pas un certain juridisme. Parmi les termes les plus galvaudés, il faut signaler les calques «minutes », « agenda », «item», «secondeur», « seconder », « hors d'ordre » et « point d'ordre».

Le mot anglais minutes, lorsqu'il est employé dans son sens strict, doit se rendre par PROCÉS-VERBAL. Le procès-verbal est un relevé officiel des délibérations. Si le rapport n'a rien d'officiel, il devient alors un simple COMPTE RENDU.

Les réunions se déroulent selon un programme adopté au début de la séance et qu'on nomme ORDRE DU JOUR, traduction correcte de l'anglais agenda. L'ordre du jour lui-même se compose de POINTS ou de QUESTIONS et non d'《 items ».

Au procès-verbal doivent figurer, pour chaque PROPOSITION (resolution), les noms du PROPOSEUR ou PREMIER PARRAIN (mover) et du SECOND PARRAIN (seconder). Habituellement, la formule adoptée est la suivante : "Sur la proposition de $M$. X, appuyé par $M$. $Y$, il est résolu que... » On ne «seconde » pas une proposition, on l'appuie.

9. Grand Larousse Encyclopédique, Paris, Larousse, Supplément II, 1975, sub verbis.

10. J. Jéraute.

11. J. Jéraute.

12. J. Jéraute.

13. J. Jéraute.

14. J. Jéraute. 


\begin{tabular}{|c|c|c|}
\hline minutes & 4 minutes > & $\begin{array}{l}\text { procès-verbal } \\
\text { compte rendu }\end{array}$ \\
\hline agenda & \& agenda > & ordre du jour \\
\hline item on the agenda & ( item sur l'agenda & $\begin{array}{l}\text { point a/de l'ordre. } \\
\text { du jour }\end{array}$ \\
\hline resolution & « résolution & $\begin{array}{l}\text { proposition } \\
\text { motion }^{15}\end{array}$ \\
\hline out of order & 4 hors d'ordre ? & $\begin{array}{l}\text { irrégulier ; irrecevable } \\
\text { contraire au règlement }\end{array}$ \\
\hline $\begin{array}{l}\text { point of order } \\
\text { mover }\end{array}$ & e point d'ordre & $\begin{array}{l}\text { appel au règlement }{ }^{16} \\
\text { proposeur } \\
\text { parrain }\end{array}$ \\
\hline move/to & & proposer \\
\hline seconder & \& secondeur \$ & second parrain \\
\hline second/to & \& seconder & appuyer \\
\hline
\end{tabular}

\subsection{Structure et exploitation de l'entreprise}

La constitution d'une entreprise exige des démarches d'ordre juridique. Son statut est également juridique. Les usages légaux étant différents selon les cultures, il est normal que ces disparités aient eu des conséquences sur la terminologie. Le premier de ces problèmes est soulevé par le mot «corporation * que nos lois ont sanctionne, mais qui n'a aucune caution de l'usage en français commun où le mot SOCIETÉ s'est implanté.

Selon son statut, une société peut être à but lucratif ou sans but lucratif, à responsabilité limitée, anonyme, etc. Nous n'entrerons pas dans ces particularités par crainte de nous y enliser. Nous retiendrons seulement que le STATUT définit la personnalité morale de l'entreprise tandis que les STATUTS en définissent les règles de fonctionnement. On dit aussi dans ce dernier sens RĖGLEMENT INTERIEUR.

C'est ce règlement qui définit l'année d'exploitation de l'entreprise, c'està-dire son EXERCICE ${ }^{17}$ et non pas 1' année fiscale > (fiscal year). L'exercice ne coincide pas toujours avec l'ANNEE CIVILE, appelée souvent à tort 1 ' * année de calendrier (calendar year).

Les entreprises où le personnel doit manipuler de fortes sommes d'argent font verser à leurs agents, en général par l'intermédiaire d'une compagnie d'assurance (bonding company), un cautionnement qui met l'entreprise à l'abri des

15. Arrêtés de terminologie de la République française.

16. Paul Robert, Dictionnaire alphabétique et analogique de la langue francaise.

17. Comité de terminologie française, Terminologie comptable, Montréal, Ordre des comptables agréés du Québec, bulletin no 4, p. 2, 1971. 
malversations. Les entreprises qui fonctionnent ainsi sont appelées bonded company, les agents qui font l'objet d'un cautionnement (bond) sont cautionnés (bonded). Si le cautionnement garantit l'exécution d'une soumission (bid bond), la soumission est dite cautionnée (bonded bid).

\begin{tabular}{|c|c|c|}
\hline corporation & «corporation & $\begin{array}{l}\text { société } \\
\text { compagnie }\end{array}$ \\
\hline status & & $\begin{array}{l}\text { statut } \\
\text { situation }\end{array}$ \\
\hline statutes. & & $\begin{array}{l}\text { statuts }{ }^{18} \\
\text { règlement intérieur }\end{array}$ \\
\hline fiscal year & " année fiscale \$ & exercice \\
\hline calendar year & " année de calendrier» & année civile \\
\hline bonding company & & $\begin{array}{l}\text { société de garantie } \\
\text { société de cautionnement }\end{array}$ \\
\hline bonded company & & société cautionnée \\
\hline bonded employee & & agent cautionné \\
\hline bond & & cautionnement \\
\hline bid bound & & $\begin{array}{l}\text { cautionnement de } \\
\text { soumission }\end{array}$ \\
\hline bonded bid & & soumission cautionnée \\
\hline
\end{tabular}

4.0 Prenons un demier volet pour l'étude de divers termes et expressions, d'origine juridique, qu'on retrouve mêlés à la terminologie courante des affaires.

Il y a d'abord les fameux latinismes de jure, de facto, ad hoc, bona fide. Ces expressions sont souvent employées telles quelles au Canada alors qu'elles n'ont pas cours dans les pays francophones, du moins pas avec la même fréquence. Les expressions de jure et de facto doivent se rendre en français par EN DROIT et EN FAIT ${ }^{19}$. L'expression ad hoc équivaut en règle générale à l'adjectif SPECIAL ${ }^{20}$.

La langue administrative d'ici fait un usage abusif de cette dernière locution, qui n'a même pas les honneurs des pages roses du Petit Larousse.

Bona fide ne figure pas non plus au palmarès des pages roses. L'expression, telle qu'elle est utilisée en anglais, a deux sens : $1^{\circ}$ de bonne foi, $2^{\circ}$ authentique. Ce sont les deux traductions qu'il faut lui substituer à la lumière du contexte. Un homme de bonne foi ; un contrat authentique.
de jure
en droit
de facto
en fait

18. Paul Robert, Dictionnaire alphabétique et analogique de la langue française.

19. J. Jéraute.

20. J. Jéraute. 
ad hoc

spécial

bona fide

de bonne foi

authentique

$S^{\prime}$ il faut continuer la lutte contre les latinismes, il convient de ne pas oublier l'affidavit qui s'applique à une DÉCLARATION SOUS SERMENT. L'expression latine n'est pas usitée dans la langue juridique française. Puisqu'on en est au serment, il faut rappeler qu'en français, on n' administre » pas un serment, mais on le fait prêter.

affidavit

déclaration sous serment

administer an oath/to *administrer un serment * faire prêter serment

Il reste enfin deux expressions, elles aussi génératrices d'anglicismes, sur lesquelles il convient de se pencher : false pretences et without prejudice.

L'expression false pretences s'applique à des affirmations fausses concernant des faits ou des événements passés en vue d'extorquer certains avantages ou privilèges. Rendue infailliblement par « fausses représentations », cette expression devrait correspondre, selon les contextes, à allégations fausses, abus de confiance, escroquerie. Exemples : Obtenir un poste par fraude; soutirer de l'argent par abus de confiance.

Employée absolument, without prejudice est une expression qu'on utilise pour réserver ses droits. Rendue littéralement, par « sans préjudice », elle n'a pas grand sens. En français, on dira selon les contextes : sous réserve des droits pertinents, sous toutes réserves ${ }^{21}$.

false pretences

allégations fausses

abus de confiance

escroquerie

without prejudice

sous réserve des droits

pertinents

sous toutes réserves

L'influence de la langue juridique anglaise est peut-être particulièrement sensible en gestion où la langue de travail dominante a toujours été l'anglais. Le travail qui précède ne fait que donner une idée de l'ampleur de cette invasion. Ici comme ailleurs, il n'y a qu'un remède à appliquer : inventorier la langue juridique française, recouper les notions et substituer les bons équivalents aux mauvais. Il n'y a pas moyen de « travailler en français » autrement.

ROBERT DUBUC

21. J. JEraute. 